\title{
Takayasu Arteritis and Ulcerative Cutaneous Sarcoidosis
}

\author{
Gangji $\mathrm{Ri}^{1}$, Emi Yoshikawa ${ }^{1}$, Tarou Shigekiyo ${ }^{1}$, Rui Ishii ${ }^{1}$, Yusuke Okamoto ${ }^{1}$, \\ Ken Kakita ${ }^{1}$, Toshihiro Otsuka ${ }^{2}$, Hideaki Morita ${ }^{1}$, Motomu Tsuji ${ }^{3}$, \\ Shinichi Moriwaki ${ }^{2}$, Masahiro Daimon ${ }^{4}$, Takahiro Katsumata ${ }^{4}$, \\ Koichi Sohmiya ${ }^{1}$, Masaaki Hoshiga ${ }^{1}$ and Nobukazu Ishizaka ${ }^{1}$
}

\begin{abstract}
A 67-year-old woman was referred to our hospital due to a refractory lower extremity ulcer. Occlusion of the bilateral superficial femoral arteries and a difference $(>50 \mathrm{mmHg})$ in blood pressure between the bilateral upper limbs were noted. In addition to occlusion of the left subclavian artery and stenosis at the ostium of the right coronary artery, these findings led to a diagnosis of Takayasu arteritis. Furthermore, a biopsy of the ulcerated skin lesion localized on the fibular surface showed a non-caseating cutaneous granulomatous lesion resulting in the diagnosis of cutaneous sarcoidosis. The simultaneous occurrence of cutaneous sarcoidosis and Takayasu arteritis, albeit rare, should not be overlooked.
\end{abstract}

Key words: Takayasu arteritis, cutaneous sarcoidosis, coronary artery stenosis, HLA-B39

(Intern Med 54: 1075-1080, 2015)

(DOI: 10.2169/internalmedicine.54.3345)

\section{Introduction}

Sarcoidosis is a multiorgan granulomatous disease, with the skin being affected in approximately $25 \%$ of cases (1). Various types of skins lesion associated with sarcoidosis have been reported, and ulcerative lesion formation, although rare, represents one of many atypical presentations (2). Several recent case reports have described the coexistence of Takayasu arteritis and immune-related disorders, including sarcoidosis (3). In this report, we describe the case of a patient with Takayasu arteritis who presented with refractory ulcerative skin lesions of the lower extremities initially caused by limb ischemia due to occlusion of the peripheral arteries; these lesions were eventually diagnosed histologically as necrotizing granulomas of cutaneous sarcoidosis.

Case Report

A 67-year-old woman who had been periodically followed up for hypothyroidism developed a refractory ulcer of the left lower limb in December 2013. Computed tomography (CT) arterial angiography showed occlusion of the bilateral superficial femoral arteries (Fig. 1A), and the patient was referred to our hospital for revascularization. She had been previously treated for hypothyroidism and exhibited a euthyroid status at referral. Her initial vital signs included a blood pressure of $145 / 80 \mathrm{mmHg}$ and heart rate of 54 beats per minute. A chest X-ray revealed mild cardiomegaly with a cardiothoracic ratio of $57 \%$, whereas an electrocardiogram was unremarkable (Fig. 1B, C), with the exception of occasional premature ventricular contractions. The ankle-brachial pressure index $(\mathrm{ABI})$ was reduced bilaterally; however, the blood pressure in the left upper limb was $55 \mathrm{mmHg}$ higher than that observed in the right upper limb (Fig. 1D). Cardiac ultrasonography showed a left ventricular ejection fraction

\footnotetext{
${ }^{1}$ Department of Thoracic and Cardiovascular Surgery, Osaka Medical College, Japan, ${ }^{2}$ Department of Dermatology, Osaka Medical College, Japan, ${ }^{3}$ Department of Pathology, Osaka Medical College, Japan and ${ }^{4}$ Department of Cardiovascular Surgery, Osaka Medical College, Japan Received for publication May 23, 2014; Accepted for publication November 5, 2014 Correspondence to Dr. Nobukazu Ishizaka, ishizaka@poh.osaka-med.ac.jp
} 

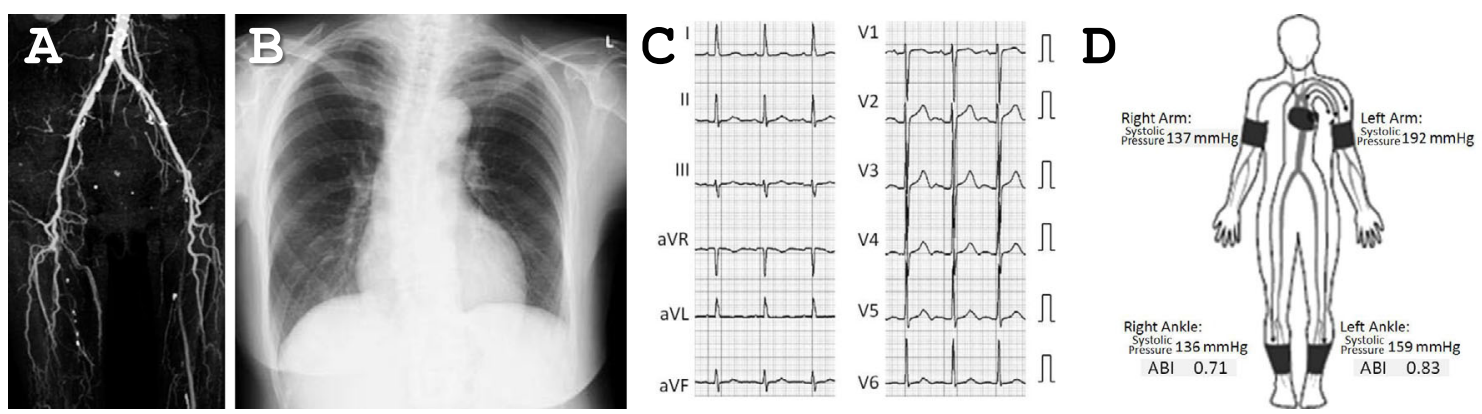

Figure 1. Lower extremity angiography, chest roentgenogram, electrocardiogram and ankle-brachial index findings obtained on admission. A: Lower extremity computed tomography angiography. The bilateral superficial femoral arteries were occluded. B: A chest roentgenogram showed mild cardiomegaly. C: No abnormalities of the ST segment were noted on an electrocardiogram. D: The ankle-brachial index (ABI) indicated a marked difference $(55 \mathrm{mmHg})$ in blood pressure between the right and left upper extremities and decreased bilateral ABI.
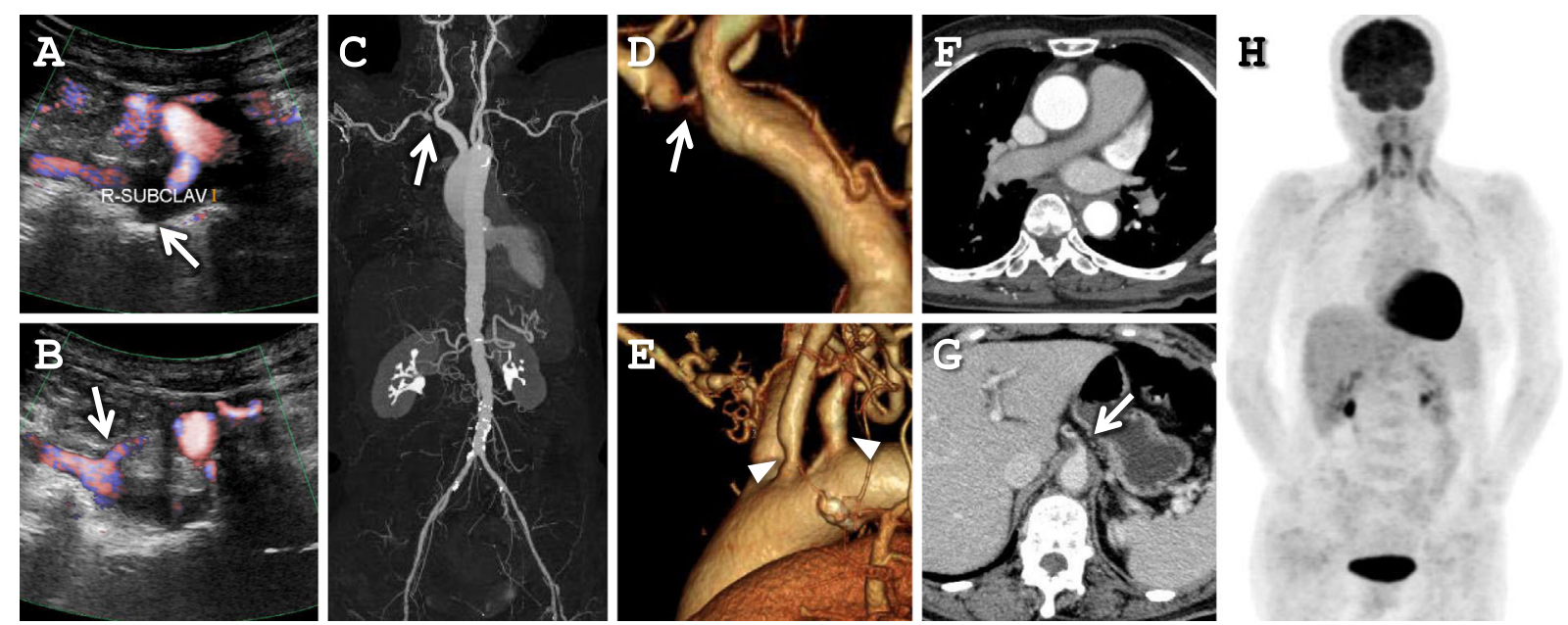

Figure 2. Ultrasonography, computed tomography (CT) and ${ }^{18}$ F-fluorodeoxyglucose positron emission tomography (FDG-PET) imaging. A, B: Ultrasonography of the neck showed occlusion of the right subclavian artery ( $A$, arrow) and the flow from the vertebral artery to the distal portion of the occluded subclavian artery (B, arrow). C-G: CT with contrast enhancement. C: Occlusion of the right subclavian artery (arrow) is observed. In the abdominal aorta, wall calcification and diffuse wall irregularities are noted. D, E: Three-dimensional reconstruction of the CT image. Severe narrowing of the proximal portion of the right subclavian artery (D, arrow) as well as moderate and mild narrowing of the proximal portion of the left common carotid artery and left subclavian artery, respectively (E, arrowheads). F, G: Horizontal planes of the CT image. Thickening of the aortic wall of the thoracic (F) and abdominal (G) aorta is observed. Luminal narrowing of the proximal portion of the celiac artery is also seen (G, arrow). F: Coronal plane image of FDG-PET. No enhanced FDG uptake is observed in the aorta or major arteries.

of $56 \%$ with no evidence of clinically problematic valvular disease, and neck ultrasonography demonstrated an interruption of the blood flow in the proximal portion of the right subclavian artery and the collateral flow from the vertebral artery to the distal portion of the right subclavian artery (Fig. 2A, B, arrows).

Ultrasonography of the lower limbs showed extensive plaque formation at the level of the bilateral iliac and common femoral arteries and occlusion of the bilateral superficial femoral arteries. Contrast-enhanced CT confirmed (sub) total occlusion of the proximal portion of the right subclav- ian artery (Fig. 2C, D), luminal narrowing of the left common carotid artery (Fig. 2E, arrowheads), wall thickening and luminal stenosis in the aorta and aortic arch branches with thickening and diffuse irregularities of the aortic wall (Fig. 2F, G) and luminal narrowing of the first branches from the aorta, including the celiac artery (Fig. 2G, arrow). ${ }^{18}$ F-fluorodeoxyglucose-positron emission tomography (FDG-PET) showed no enhanced uptake in the aorta or major arteries (Fig. 2H).

Adenosine triphosphate-stress myocardial perfusion imaging with thallium-201 showed decreased, albeit reversible, 

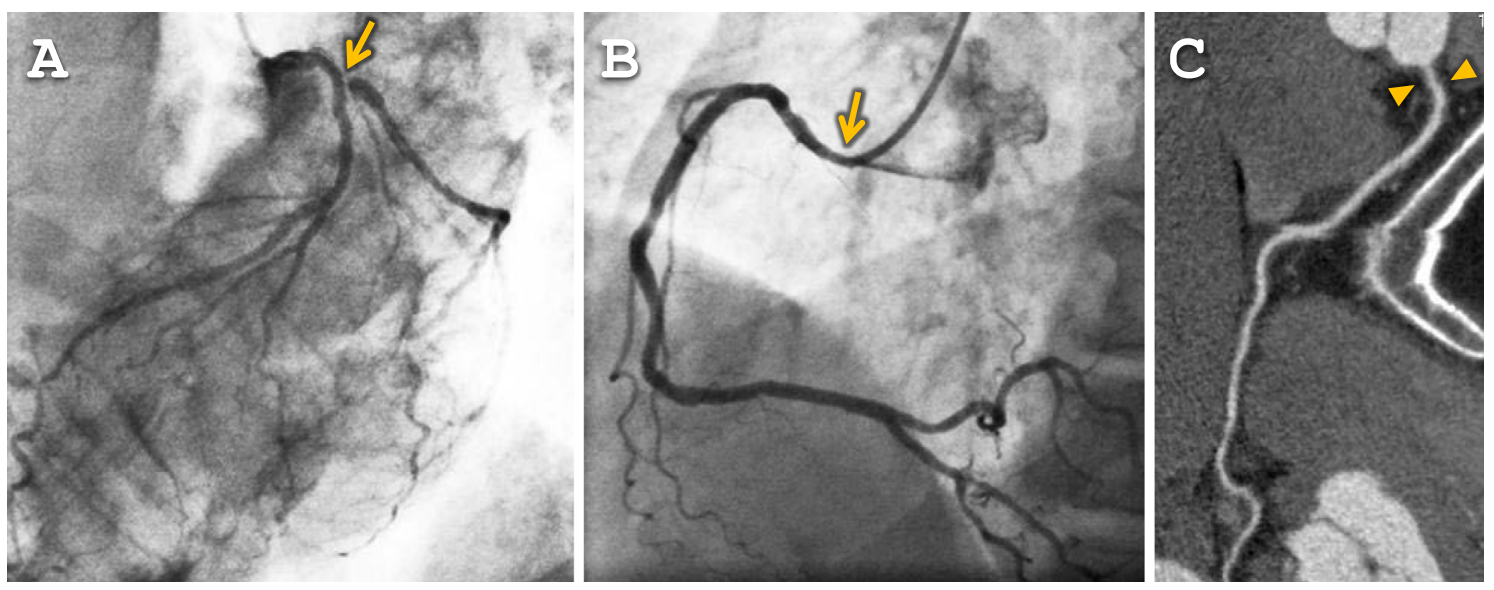

Figure 3. Invasive and computed tomography (CT) coronary angiography. A, B: Coronary artery angiography. C: CT angiography. The proximal portion of the left circumflex artery showed subtotal occlusion (A, arrow), while the ostial portion of the right coronary artery exhibited stenosis (B, arrow) accompanied by coronary artery wall thickening illustrated on $\mathrm{CT}$ angiography (C, arrowheads).
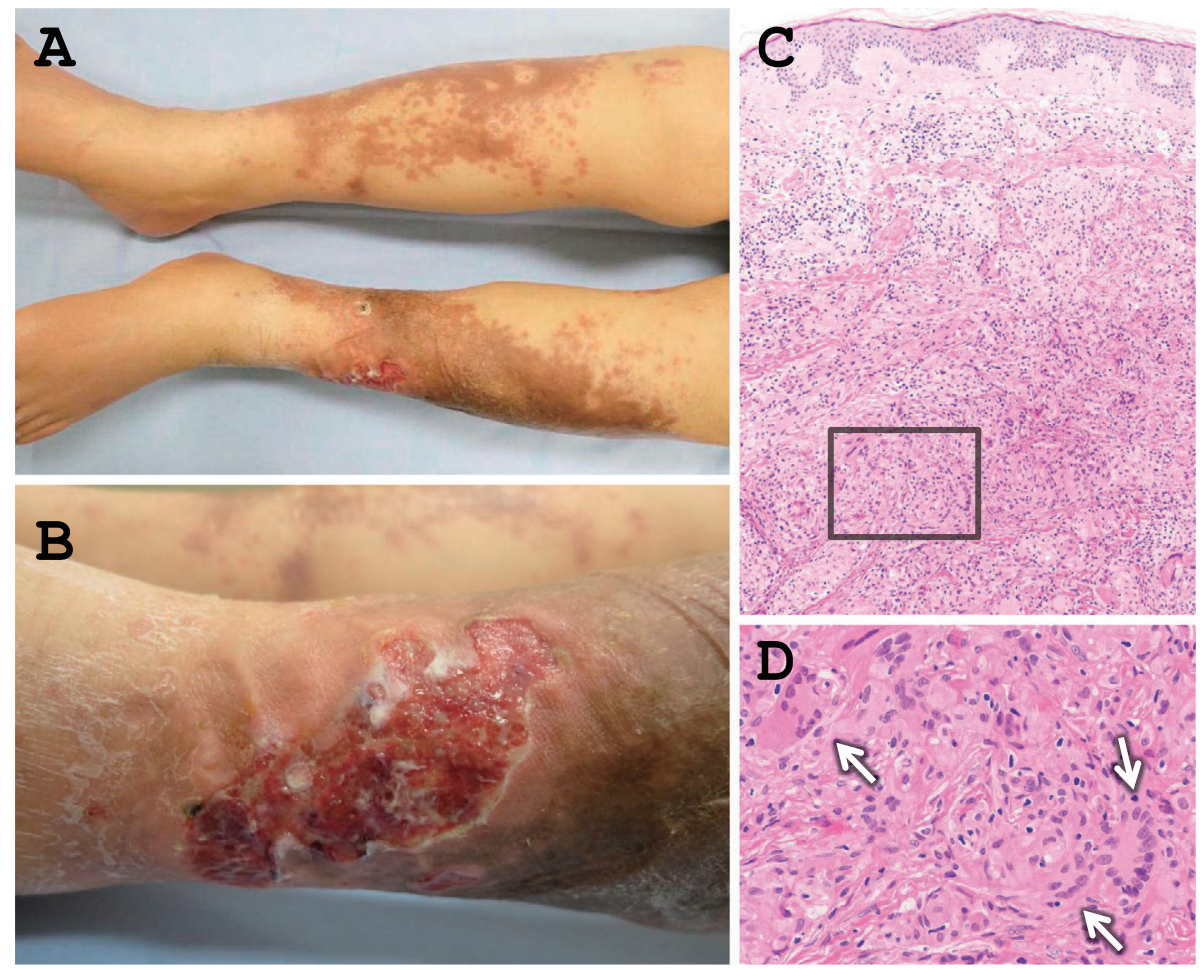

Figure 4. Clinical appearance of the indurated, coalescent pigmented macules and plaques of variable sizes on the bilateral lower extremities. A, B: Photographs of the lower extremities. A fist-sized gangrenous skin ulcer developed on the left lateral malleolus (B). C, D: Hematoxylin and Eosin staining of a biopsy specimen of the pigmented macules showed dermal inflammatory cell infiltration at low $(C$, original magnification $\times 40)$ and high $(D$, original magnification $\times 200)$ magnification. Multinucleated giant cells in multiple discrete non-necrotizing epithelioid granulomas were observed in the deep dermis ( $\mathrm{D}$, arrows).

nuclear accumulation in the anterior, inferior and posterior walls (data not shown). Coronary artery angiography showed $75 \%$ stenosis in the ostial region of the right coronary artery and severe stenosis in the ostial region of the left circumflex artery (Fig. 3). Histology of biopsy specimens of the pigmented skin lesions on the lower limbs (Fig. 4A, B) showed prominent granulocytic and lymphocytic infiltration, which was also present in the periarterial regions, in addition to non-caseating epithelioid cell granuloma with multinucleated giant cells without vasculitis, leading to a diagno- 
sis of cutaneous sarcoidosis (Fig. 4C, D arrows).

Laboratory studies showed a white blood cell (WBC) count of $3,460 / \mu \mathrm{L}$, hemoglobin level of $11.3 \mathrm{~g} / \mathrm{dL}$, platelet count of $14.8 \times 10^{4} / \mu \mathrm{L}$, serum $\mathrm{C}$-reactive protein concentration of $0.55 \mathrm{mg} / \mathrm{dL}$, serum soluble interleukin-2 receptor level of $787 \mathrm{U} / \mathrm{mL}$ and erythrocyte sedimentation rate of 36 $\mathrm{mm} / \mathrm{hr}$. The plasma B-type natriuretic peptide level was mildly elevated at $115.3 \mathrm{pg} / \mathrm{mL}$. Syphilis tests were negative and the ACE level was $10.5 \mathrm{U} / \mathrm{L}$. Meanwhile, the serum corrected calcium level was $9.0 \mathrm{mg} / \mathrm{dL}$ and the phosphorus level was $3.8 \mathrm{mg} / \mathrm{dL}$. Tests for proteinase 3 anti-neutrophil cytoplasmic antibodies (ANCA) and myeloperoxidasespecific ANCA were negative, and a genetic examination for human leukocyte antigen (HLA) typing showed positivity for HLA-B39.

The patient underwent coronary bypass surgery-saphenous vein grafting of the right coronary artery and left internal thoracic artery to the left circumflex artery. Histology of the aortic wall specimen showed mild mucous degeneration without apparent infiltration of inflammatory cells. The Takayasu arteritis activity was considered to be low based on the findings of FDG-PET, and the lower extremity lesions were treated with topical corticosteroid ointment.

\section{Discussion}

We herein reported the case of a patient with coronary and peripheral arterial stenosis or occlusion, aortic and arterial wall thickening and skin lesions with ulcer formation in the lower extremities. Although the possibility of arteriosclerosis obliterans was first suspected, the localization and appearance of the skin lesions differed from that of severe ischemia. The detection of a marked difference $(>50 \mathrm{mmHg})$ in blood pressure between the right and left upper limbs, coupled with the stenosis of the aortic arch branches and first aortic branches, led to a diagnosis of Takayasu arteritis. In addition, the lower limb skin lesion was diagnosed as cutaneous sarcoidosis and ulcerative vasculitis.

There is some debate over the diagnosis of Takayasu arteritis in our patient. Based on the aortic wall sample obtained at the time of coronary bypass surgery, we diagnosed him with Takayasu arteritis in accordance with the 1990 Criteria for the Classification of Takayasu Arteritis advocated by the American College of Cardiology (ACR). Among six ACR criteria for the diagnosis of Takayasu arteritis, our patient met three items: claudication of the extremities, a difference in blood pressure $(>10 \mathrm{mmHg}$ difference in systolic blood pressure between the arms) and arteriogram abnormalities (narrowing of the primary branches of the aorta). Another criterion, the presence of a bruit over the subclavian arteries or aorta, may also have been fulfilled, considering the detection of occlusion of the left subclavian artery and the shunt flow from the left common carotid artery. A fundoscopic examination revealed no abnormal findings. Among the ACR diagnostic criteria, an 'age of onset of less than 40 years' was not fulfilled in this case. On the other hand, FDG-PET showed that Takayasu arteritis, if present, was not in the active stage. In addition, among a US cohort of 126 patients with Takayasu arteritis, Schmidt et al. reported that 31 patients $(25 \%)$ were 40 years of age or older at diagnosis (4). An increase in the levels of CD8-positive subsets has been reported in cases of Takayasu arteritis (5), and an elevated $\mathrm{CD}^{+} / \mathrm{CD}^{+} \mathrm{T}$-cell ratio and bronchoalveolar lavage lymphocytosis, in addition to an increased serum ACE level, are findings supporting the diagnosis of sarcoidosis (6), although T-cell subsets (CD4 and CD8) were not assessed in the current case.

The patient exhibited other clinical features that may be associated with Takayasu arteritis. First, DNA typing of the HLA genes showed that he had HLA-B39. Several HLA types have been reported to be associated with Takayasu arteritis, among which HLA-B52 may be the most common. For example, the HLA-B39 allele has been shown to be associated with Takayasu arteritis in Asian populations $(7,8)$, although there is some controversy regarding this finding (9). The presence of the HLA-B39 allele is another finding supporting a diagnosis of Takayasu arteritis according to the Japanese diagnostic guidelines (Japanese Circulation Society 2008) (10). Based on these criteria, thickening of the thoracic aortic wall, as seen in our patient, is considered to be a characteristic finding on diagnostic imaging.

Second, our patient had coronary artery lesions in the proximal portion of the left circumflex artery. Coronary artery lesions have been reported to be present in approximately $10 \%$ to $30 \%$ of patients with Takayasu arteritis (11). Using coronary angiography to assess 81 patients with Takayasu arteritis, Endo et al. found that 24 subjects had coronary stenosis (12). The ostium was most frequently affected in 21 patients $(87.5 \%)$, while the proximal portion of the coronary artery was affected in the remaining three patients $(12.5 \%)$. More recently, Sun et al. showed that, among 487 patients with Takayasu arteritis, $45(7.7 \%)$ had coronary artery lesions, with the ostia $(37.4 \%)$ and proximal segments $(33.3 \%)$ of the coronary artery being most frequently involved (13).

There is further debate over whether the coexistence of sarcoidosis and Takayasu arteritis is incidental. Weiler et al. described eight cases involving the simultaneous occurrence of sarcoidosis and vasculitis, five patients of which were diagnosed with Takayasu arteritis, in a review of the literature published in 2000 (14). Furthermore, Hamzoui et al. described three other cases in 2011, published as a case report with a literature review (3). More recently, Izumikawa et al. presented the case of a 24-year-old man with Crohn's disease who presented with narrowing of the abdominal aorta and proximal subclavian artery in addition to stenosis of the left renal artery, leading to a diagnosis of Takayasu arteritis. That patient exhibited swelling of the bilateral hilar and mediastinal lymph nodes, and sarcoidosis was diagnosed according to the histology of the lung tissues and mediastinal lymph nodes (15). Notably, Bilge et al. reported that some patients with Takayasu arteritis have immune-related disor- 
ders, including sarcoidosis, systemic lupus erythematosus, rheumatoid arthritis and sarcoidosis, based on their medical history, although the prevalence of these conditions was not reported (16). Fewer reports have demonstrated the occurrence of cutaneous sarcoidosis and Takayasu arteritis, although Varus et al. reported the case of a man with a 10year history of cutaneous and pulmonary sarcoidosis who developed Takayasu arteritis that subsequently induced ischemia of the right upper limb (17).

Ulcerative cutaneous sarcoidosis is a form of cutaneous sarcoidosis $(2,18)$. The cutaneous findings of ulcerative sarcoidosis may resemble those of necrobiosis lipoidica, an idiopathic, chronic granulomatous inflammatory disorder involving collagen degeneration leading to skin atrophy. Patients with necrobiosis lipoidica exhibit infiltration of lymphoplasma cells surrounding the blood vessels, possibly associated with wall thickening; however, these findings were not observed in the current case (19). Arteriosclerosis obliterans, a cutaneous ulcer, may also develop, although these lesions may initially form on the heel or the top of toe in the lower extremities $(20,21)$. Such lesions were, again, absent in the current case.

Several previous reports have presented cases of sarcoid granulomatous vasculitis (22). Fernandes et al. pointed out that systemic vasculitis may occur as a complication, albeit unusual, of sarcoidosis (23). They subsequently reported six patients with sarcoidosis and systemic vasculitis and discussed the possibility that sarcoid vasculitis may mimic other types of vasculitis, including Takayasu arteritis. In their series, vasculitis was not diagnosed based on histology, but rather arteriography. More recently, Ward et al. documented the case of a patient with subcutaneous sarcoidosis who may have eventually developed epicardial coronary artery involvement, as suspected on computed tomographic images (24). In the current case, the skin biopsy showed granulomatous lesion formation, although fibrinoid degeneration of the vessels was not present; therefore, sarcoid vasculitis was not diagnosed according to the cutaneous lesion. Although non-caseating granulomata lesion formation in the coronary arteries may occur in patients with sarcoidosis $(25,26)$, we do not believe that sarcoidosis vasculitis would explain the coronary artery lesions observed in our patient given the absence of epicardial thickening.

In summary, we herein described the case of a patient with multiple vascular lesions suggestive of Takayasu arteritis. Although the blood flow to the lower extremities was decreased based on the ABI, the ulcerative lesions were considered to be due to cutaneous sarcoidosis. Physicians must be aware of the concomitant occurrence of (presumable) immune-related disorders in patients with Takayasu arteritis and conversely those with sarcoidosis.

The authors state that they have no Conflict of Interest (COI).

\section{References}

1. Noiles K, Beleznay K, Crawford RI, et al. Sarcoidosis can present with necrotizing granulomas histologically: two cases of ulcerated sarcoidosis and review of the literature. J Cutan Med Surg 17: 377-383, 2013.

2. Ichiki Y, Kitajima Y. Ulcerative sarcoidosis: case report and review of the Japanese literature. Acta Derm Venereol 88: 526-528, 2008.

3. Hamzaoui A, Salem R, Klii R, et al. Co-existing sarcoidosis and Takayasu arteritis: report of a case. Int Arch Med 4: 9, 2011.

4. Schmidt J, Kermani TA, Bacani AK, et al. Diagnostic features, treatment, and outcomes of Takayasu arteritis in a US cohort of 126 patients. Mayo Clin Proc 88: 822-830, 2013.

5. Uppal SS, Verma S. Analysis of the clinical profile, autoimmune phenomena and T cell subsets (CD4 and CD8) in Takayasu's arteritis: a hospital-based study. Clin Exp Rheumatol 21: S112S116, 2003.

6. Hyldgaard C, Kaae S, Riddervold M, et al. Value of s-ACE, BAL lymphocytosis, and CD4+/CD8+ and CD103+CD4+/CD4+ T-cell ratios in diagnosis of sarcoidosis. Eur Respir J 39: 1037-1039, 2012.

7. Kimura A, Kitamura H, Date Y, et al. Comprehensive analysis of HLA genes in Takayasu arteritis in Japan. Int J Cardiol 54 (Suppl): S61-S69, 1996.

8. Kitamura H, Kobayashi Y, Kimura A, et al. Association of clinical manifestations with HLA-B alleles in Takayasu arteritis. Int J Cardiol 66 (Suppl 1): S121-S126, 1998.

9. Takamura C, Ohhigashi H, Ebana Y, et al. New human leukocyte antigen risk allele in Japanese patients with Takayasu arteritis. Circ J 76: 1697-1702, 2012.

10. Japanese Circulation Society. Guideline for management of vasculitis syndrome (JCS 2008). Circ J 75: 474-503, 2011.

11. Amano J, Suzuki A. Coronary artery involvement in Takayasu's arteritis. Collective review and guideline for surgical treatment. J Thorac Cardiovasc Surg 102: 554-560, 1991.

12. Endo M, Tomizawa $Y$, Nishida $H$, et al. Angiographic findings and surgical treatments of coronary artery involvement in Takayasu arteritis. J Thorac Cardiovasc Surg 125: 570-577, 2003.

13. Sun $\mathrm{T}$, Zhang H, Ma W, et al. Coronary artery involvement in Takayasu arteritis in 45 Chinese patients. J Rheumatol 40: 493497, 2013.

14. Weiler V, Redtenbacher S, Bancher C, et al. Concurrence of sarcoidosis and aortitis: case report and review of the literature. Ann Rheum Dis 59: 850-853, 2000.

15. Izumikawa $\mathrm{K}$, Motoi $\mathrm{N}$, Takaya $\mathrm{H}$, et al. A case of concurrent sarcoidosis, aortitis syndrome and Crohn's disease. Intern Med 50: 2915-2917, 2011.

16. Bilge NS, Kasifoglu T, Cansu DU, et al. Retrospective evaluation of 22 patients with Takayasu's arteritis. Rheumatol Int 32: 11551159, 2012.

17. Vaurs C, Ammoury A, Cordel N, et al. Large-vessel granulomatous vasculitis during the course of sarcoidosis: Takayasu's arteritis? Ann Dermatol Venereol 136: 890-893, 2009 (in French, Abstract in English).

18. Hunt RD, Gonzalez ME, Robinson M, et al. Ulcerative sarcoidosis. Dermatol Online J 18: 29, 2012.

19. Patrascu V, Giurca C, Ciurea RN, et al. Ulcerated necrobiosis lipoidica to a teenager with diabetes mellitus and obesity. Rom J Morphol Embryol 55: 171-176, 2014.

20. Sugihara S, Yamamoto $Y$, Matsubara K, et al. Autoperipheral blood mononuclear cell transplantation improved giant ulcers due to chronic arteriosclerosis obliterans. Heart Vessels 21: 258-262, 2006.

21. Hanafusa T, Yamaguchi Y, Katayama I. Intractable wounds caused by arteriosclerosis obliterans with end-stage renal disease treated by aggressive debridement and epidermal grafting. J Am Acad 
Dermatol 57: 322-326, 2007

22. Ishii A, Hoshii Y, Nakashima T, et al. Sarcoidosis with pulmonary hypertension exacerbated by Takayasu-like large vessel vasculitis. Pathol Int 61: 546-550, 2011.

23. Fernandes SR, Singsen BH, Hoffman GS. Sarcoidosis and systemic vasculitis. Semin Arthritis Rheum 30: 33-46, 2000.

24. Ward EV, Nazari J, Edelman RR. Coronary artery vasculitis as a presentation of cardiac sarcoidosis. Circulation 125: e344-e346,
2012.

25. Butany J, Bahl NE, Morales K, et al. The intricacies of cardiac sarcoidosis: a case report involving the coronary arteries and a review of the literature. Cardiovasc Pathol 15: 222-227, 2006.

26. Lam CS, Tolep KA, Metke MP, et al. Coronary sarcoidosis presenting as acute coronary syndrome. Clin Cardiol 32: E68-E71, 2009.

(C) 2015 The Japanese Society of Internal Medicine http://www.naika.or.jp/imonline/index.html 